\title{
Increased RAB31 Expression in Cancer-Associated Fibroblasts Promotes Colon Cancer Progression Through HGF-MET Signaling
}

\author{
Tang Yang ${ }^{1 \dagger}$, Huang Zhiheng ${ }^{1,2 \dagger}$, Wang Zhanhuai ${ }^{1}$, Xiao Qian $^{1}$, Liu Yue ${ }^{1}$, Ge Xiaoxu ${ }^{1}$, \\ Wei Jingsun ${ }^{1}$, Zheng Shu ${ }^{1}$ and Ding Kefeng ${ }^{1 *}$ \\ ${ }^{1}$ Key Laboratory of Cancer Prevention and Intervention, Ministry of Education, Department of Colorectal Surgery and \\ Oncology, The Second Affiliated Hospital, Zhejiang University School of Medicine, Hangzhou, China, ${ }^{2}$ Department of \\ Otorhinolaryngology, The Second Affiliated Hospital of Zhejiang University School of Medicine, Hangzhou, China
}

OPEN ACCESS

Edited by:

Cornelis F. M. Sier,

Leiden University, Netherlands

Reviewed by:

Lukas Hawinkels,

Leiden University Medical

Center, Netherlands

Hugo de Jonge

University of Pavia, Italy

Cristiana Angelucci,

Catholic University of the Sacred

Heart, Rome, Italy

${ }^{*}$ Correspondence:

Ding Kefeng

dingkefeng@zju.edu.cn

†These authors have contributed equally to this work

Specialty section:

This article was submitted to Gastrointestinal Cancers,

a section of the journal

Frontiers in Oncology

Received: 26 May 2020

Accepted: 04 August 2020

Published: 23 September 2020

Citation:

Yang $T$, Zhiheng $H$, Zhanhuai $W$, Qian X, Yue L, Xiaoxu G, Jingsun W, Shu $Z$ and Kefeng D (2020) Increased

RAB31 Expression in Cancer-Associated Fibroblasts Promotes Colon Cancer Progression

Through HGF-MET Signaling.

Front. Oncol. 10:1747.

doi: 10.3389/fonc. 2020.01747
RAB family proteins participate in the dynamic regulation of cellular membrane compartments and are dysregulated in a variety of tumor types, which may alter the biological properties of cancer cells such as proliferation, migration, and invasion. In our previous study, we found that Ras-related protein Rab-31 (RAB31) expression was increased in late-stage colorectal cancer (CRC). The role of RAB31 has never been investigated in CRC. In this study, we found that expression of RAB31 in the tumor stroma but not cancer cells of colon cancer predicted poor survival. RAB31 can be detected in primary cancer-associated fibroblasts (CAFs) and paired normal fibroblasts. Conditioned medium (CM) from RAB31 overexpressing CAFs significantly promoted migration of colon cancer cell lines in vitro and in vivo. This process may be mediated by paracrine action of hepatocyte growth factor (HGF), which was increased in the CM of RAB31-overexpressing CAFs. Blockade of HGF/MET signaling by drug inhibition, knockdown of mesenchymal to epithelial transition factor (MET) in RKO, or antibody neutralization of HGF abolished migration of RKO cells mediated by RAB31 expression in CAFs. We propose that in colon cancer, increased RAB31 expression in CAFs may contribute to tumor progression by regulating the secretion of HGF in the tumor stroma.

Keywords: colon cancer, cancer associated fibroblast (CAF), hepatocyte growth factor (HGF), cancer progression, Ras-related protein RAB31

\section{INTRODUCTION}

Colorectal cancer (CRC) is the third most common malignant neoplasm worldwide with over 1 million cases diagnosed every year (1). Over the last decade, with advances in sequencing techniques, huge efforts have been made in unraveling the molecular complexity behind CRC initiation and progression. In our earlier study, we combined ITRAQ mass spectrometry with gene microarray to screen for differentially expressed genes associated with CRC progression. RAB31 was found to be upregulated in stage IV CRC (2); however, its role in CRC progression has never been investigated.

RAB31 is a member of the RAB family proteins, an important subgroup of the GTPase superfamily $(3,4)$ which participates in the dynamic regulation of cellular membrane compartments and is implicated in membrane trafficking, Golgi complex organization, and the 
sorting and delivery of secretory/membrane proteins (5). RAB31 is primarily localized in the Golgi complex and can be found in endosomes and tubulovesicular structures originating from the Golgi complex (6). RAB31 has been reported to mediate the transport of the cation-dependent mannose-6-phosphate (CDMPR) from the trans-Golgi network (TGN) to endosomes (7). The underlying mechanism may involve interaction between RAB31 and the Lowe oculocerebrorenal syndrome protein OCRL-1 (an inositol polyphosphate 5-phosphatase), which is required for TGN organization and transport carrier formation (8). Accumulating evidence has shown that altered RAB protein expression is associated with cancer progression (9). RAB31 has been reported to be associated with the malignant behavior of breast cancer, hepatocellular carcinoma, and gastric cancer (1012). Increased RAB31 expression is significantly associated with distant metastasis-free survival and overall survival rate in breast cancer (13). Breast cancer cell lines with RAB31 overexpression in vitro switch from an invasive to a more proliferative phenotype (10), suggesting a pro-tumorigenic role for this protein in the respective cancer types.

While the molecular biology of cancer cells has been extensively studied, the tumor microenvironment (TME) also plays an important role in tumor progression. The TME, also known as the tumor stroma, consists of connective tissue and a variety of non-cancer cell types. Immune cells such as macrophages, cancer-associated fibroblasts (CAFs), and lymphocytes residing in the stroma have been shown to exhibit bidirectional effects on tumor progression (14-16). The tumor stroma is associated with prognosis in a variety of cancer types (17-21). In CRC, the tumor-stroma ratio predicts recurrence in patients treated with neoadjuvant chemotherapy $(22,23)$ and may serve as a prognosis factor in stage II CRC (24). According to the consensus molecular subtypes of classification for CRC (CMS classification), the CMS4 group, defined as a subtype of CRC with increased CAF component and mesenchymal activation, features higher resistance to chemotherapy and antiEGFR therapy and has the worst prognosis (25). An important mechanism underlying TME-mediated tumor progression is through paracrine action. CAF is a heterogeneous group of fibroblast-like cells and a major component of the TME stromal cells. CAF secretion of growth factors such as EGF, FGF, HGF, TGF- $\beta$, and cytokines such as IL6 facilitates the migration of cancer cells and confers resistance to antitumor therapy $(26,27)$. CAFs can also promote tumor invasion and metastasis through physical contact (28). The pro-tumor functions of other stromal cells have also been widely studied $(29,30)$.

Since high-throughput screening based on bulk tissue does not discriminate cancer cells from stromal cells, further study is needed for better interpretation of the acquired results from our previous study. In this study, we aim to characterize the functional and clinical roles of RAB31 in the progression of colon cancer. We found that while RAB31 may be expressed in both cancer cells and stromal cells, stromal RAB31 expression is associated with poor prognosis in colon cancer. Overexpression of RAB31 in CAFs promoted colon cancer cell migration in an HGF/MET-dependent manner, suggesting a role for CAF-expressed RAB31 in the migration of colon cancer cells through regulating paracrine secretion of HGF. These results provide novel information on the role of RAB31 in colon cancer progression.

\section{MATERIALS AND METHODS}

\section{Patients}

Paraffin-embedded colon tumor tissue samples were collected from the Second Affiliated Hospital of Zhejiang University, School of Medicine. A cohort of 98 patients receiving surgery from 2006 to 2007 was included. Patients who received neoadjuvant chemotherapy or incomplete surgical resection were excluded from the cohort. The diagnosis of adenocarcinoma in the patients was confirmed by two pathologists. One millimeter-diameter cores from each tumor were taken from the formalin-fixed paraffin-embedded tissues for the generation of tissue microarray.

\section{CMS Classification}

Data for analysis of RAB31 expression and CMS classification including normalized gene expression data, CMS subtyping calls, and sample annotation were readily provided and downloaded through the Synapse platform (doi: 10.7303/syn2623706) (25). The data was sorted in Microsoft Excel, and statistical analysis and plotting were performed in GraphPad Prism 8.

\section{Immunohistochemistry}

Paraffin-embedded tissues were collected to generate a tissue microarray. Immunohistochemistry was performed on $5-\mu \mathrm{m}$ thick sections. Briefly, the microarray sections were dewaxed in an oven at $60^{\circ} \mathrm{C}$, deparaffinized in xylene, and rehydrated in graded alcohol. Heated TRIS-EDTA was used for antigen retrieval. Five percentage BSA was used to block non-specific binding. The slides were stained with primary antibodies in $5 \%$ BSA at dilution of 1:200, rinsed in PBS three times, and incubated with secondary fluorescent antibodies followed by 3 more rinses in PBS. Assessments were independently conducted by 2 pathologists. The expression of RAB31 was graded in both the tumor cell and tumor stromal compartments using a 3tier scale: 1 , no staining or weak staining; 2 , moderate staining; 3 , strong staining. The results were then divided into negative staining, grade 1; and positive staining, grade 2 and 3 for further statistical analysis.

For immunostaining of cells, fibroblasts were culture on round glass coverslips in 12-well culture plates. Before staining, the coverslips were rinsed in PBS 3 times and fixed with $4 \%$ paraformaldehyde for $15 \mathrm{~min}$. The following blocking and staining procedures were the same as described above.

\section{Cell Culture}

All CRC cell lines were purchased from ATCC. HCT116 and HT29 were maintained in McCoy's 5A modified medium; RKO and DLD1 were maintained in RPMI-1640 medium; SW620 and SW480 were maintained in Leibovitz L-15 medium; LOVO, T84, and CACO2 were maintained in Dulbecco's Modified Eagle Medium. All culture mediums were supplemented with 10\% fetal bovine serum (FBS, Gibco/Invitrogen, Carlsbad, CA, USA). 
SW480 and SW620 were cultured in a $\mathrm{CO}_{2}$-free incubator, while all other cells were maintained in a humidified incubator with $95 \%$ air and $5 \% \mathrm{CO}_{2}$. The culture medium was refreshed every 3 days, and the cells were passed at $90 \%$ confluency.

\section{Preparation of Primary Fibroblast Cells and Fibroblast Conditioned Medium (CM)}

Mucosa from tumor and adjacent normal tissue were obtained from freshly surgically resected samples and transferred to the lab in PBS containing 10\% Povidone iodine within $30 \mathrm{~min}$ for further procedures. The tissues were rinsed in PBS containing $500 \mathrm{U} / \mathrm{ml}$ streptomycin and penicillin for 3 times, minced with surgical scissors into $2-4-\mathrm{mm}^{3}$ chunks, and plated in a $60-\mathrm{mm}$ culture dish in RPMI 1640 containing 10\% FBS, $100 \mathrm{U} / \mathrm{ml}$ streptomycin, and penicillin and $2.5 \mu \mathrm{g} / \mathrm{ml}$ Amphotericin B. To ensure adherence to the culture dish, the culture medium must not submerge the tissue blocks. The primary cultures were incubated at $37^{\circ} \mathrm{C}$ with $5 \% \mathrm{CO}_{2}$ and the culture medium was refreshed every 3-4 days. One to 3 weeks after plating, the proliferating fibroblast population could be observed near the minced tissue. The primary fibroblasts were then passed, and the remnant tissues were discarded. The expression of vimentin and $\alpha$-SMA and absence of CK were used to confirm the identity of normal or CAFs. Most primary fibroblasts undergo senescence after $10-15$ continuous passages, characterized by increased size and decreased proliferation rate.

For the generation of CM, fibroblasts were seeded in 24well culture plates at 50,000 cells per well. When the cells reached $80 \%$ confluency, the culture medium was replaced with RPMI-1640 containing FBS (2\% for WB-related experiments and $6 \%$ for transwell-related experiments). Three to 4 days later, the culture medium was harvested and centrifuged at $10,000 \mathrm{~g}$ for $5 \mathrm{~min}$; the supernatant was collected as fibroblast CM for subsequent experiments.

\section{Lentiviral Plasmid Construction}

RAB31 cDNA (Youbio, G118068, Hunan, China) was used as template, and $\mathrm{XhoI}$ and BamHI restriction sites were introduced into the cDNA using PCR. RAB31 was inserted to a 2ndgeneration lentiviral vector containing a CMV promotor with EGFP reporter gene (pLV-EGFP-2A, VL3401, Inovogen Tech, Beijing, China). For gene knockdown, reverse complementary ssDNA oligos were annealed in M buffer (Clontech, Mountain View, CA, USA) using a gradient cooling PCR program. The resulting dsDNA was inserted into a 2nd-generation lentiviral vector containing a U6 promotor and an EGFP reporter gene (pLVshRNA-EGFP, VL3101, Inovogen Tech, Beijing, China) using a homologous recombination kit (\#10911ES25, Yeason, Shanghai, China). Lentivirus packaging plasmids pSPAX2 (VT1444) and pMD2.G (VT1443) were purchased from Youbio, China. Primers used for qPCR and Oligos used for knockdown are included in Supplementary Tables 1, 2, respectively.

\section{Lentivirus Production and Infection}

LentiX-293T (Clontech, Mountain View, CA, USA) was grown in a $100-\mathrm{mm}$ culture dish with $12 \mathrm{ml}$ DMEM containing $10 \%$ FBS and $100 \mathrm{U} / \mathrm{ml}$ streptomycin and penicillin. At $80 \%$ confluency, a total of $25 \mu \mathrm{g}$ DNA including lentiviral vector, pSPAX2, and pMD2.G packaging vectors were transfected into the $293 \mathrm{~T}$ cells using Lipofectamine 2000 transfection reagent (Thermo Fisher Scientific, Waltham, MA, USA) at a ratio of 2:2:1. The supernatant was collected at $36 \mathrm{~h}$ and $48 \mathrm{~h}$ and filtered through a $45-\mu \mathrm{m}$ filter. The virus was concentrated using a sterilized $5 \times$ concentration reagent $[8.766 \mathrm{~g} \mathrm{NaCl}, 50 \mathrm{mg}$ PEG 8000 (SigmaAldrich, St. Louis, MO, USA) in $200 \mathrm{ml}$ Milli-Q water] which was added to the supernatant and mixed overnight and centrifuged at $4,000 \mathrm{~g}$ for $30 \mathrm{~min}$ at $4^{\circ} \mathrm{C}$. The precipitated sediment was dissolved in Opti-MEM for $100 \times$ concentration.

For lentivirus infection, fibroblasts were seeded in 6-well plates at 300,000 cells per well. Eighty microliter concentrated lentivirus was added to each well and incubated overnight before refreshing the culture medium. Three days after transfection, the cells were observed under a fluorescence microscope for EGFP expression and western blotting was used for the validation of overexpression or knockdown efficiency. Stably transfected CAFs were denoted CAF EGFP for vector control, CAF RAB31 for RAB31 overexpression, and CAF RAB31 KD for RAB31 knockdown. Similarly, RKO MET KD referred to knockdown of MET in RKO.

\section{Transwell Assay}

Transwell assay was performed using $8-\mu \mathrm{m}, 24$-well plates (Corning Costar, Lowell, MA, USA). Thirty thousand cells suspended in $200 \mu \mathrm{l}$ RPMI-1640 supplemented with 6\% FBS were seeded in the upper chamber. The lower chamber contained RPMI-1640/6\% FBS mixed with fibroblast-CM at a ratio of 2:1 or with cytokines at a concentration of $50 \mathrm{ng} / \mathrm{ml}$. For HGF neutralization experiments, the anti-HGF polyclonal antibody (AF-294-NA, R\&D Systems, Minneapolis, MNB, USA) was added to the mixed medium and incubated at $37^{\circ} \mathrm{C}$ for $1 \mathrm{~h}$ before adding to the lower chamber. For MET drug blockade, the inhibitors were added to the cell suspension in the upper chamber and incubated for $20 \mathrm{~min}$ at $37^{\circ} \mathrm{C}$ and to the mixed medium in the lower chamber right before the transwell was inserted. The transwell was incubated at $37^{\circ} \mathrm{C}$ under $5 \% \mathrm{CO}_{2}$ for 45 or $50 \mathrm{~h}$. Then, the upper chamber was fixed with $4 \%$ PFA, the upper surface of the filter was wiped with a cotton swab to remove the remaining cells, and the lower surface was stained using hematoxylin-eosin or Crystal Violet. After rinsing in water, the cells were observed under a light microscopy. The number of cells was counted in three to five random fields per transwell in which the number of cells were counted manually.

\section{Wound Healing Assay}

Cells were seeded into a silica gel mold containing two rectangular chambers divided by a septal across the middle. The mold was placed in 6-well culture dishes with each chamber containing $\sim 30,000$ cells. The cells were allowed to settle overnight before retrieving the mold (at $0 \mathrm{~h}$ ) to leave two groups of cells of $80 \%$ confluency separated by a gap. The cells were cultured in a complete culture medium supplemented with CAF $\mathrm{CM}$ for $24 \mathrm{~h}$. The area of the gap at 0 and $24 \mathrm{~h}$ was measured using the ImageJ software. The migration speed was indicated by 
a migration index calculated by the following formula:

$$
\text { migration index }=\frac{\operatorname{AREA}(0 \mathrm{~h})-\operatorname{AREA}(24 \mathrm{~h})}{\operatorname{AREA}(0 \mathrm{~h})}
$$

A larger migration index indicates faster migration speed.

\section{Western Blot Analysis (WB)}

$\mathrm{WB}$ was performed using conventional WB procedures. Primary antibodies were purchased from CST (Cell Signaling Technology, Danvers, MA, USA), including anti-HGF anti-phospho Met (\#3133), anti-cMet (\#8198), anti-Akt (\#2920), anti-phosphoAkt (ser473, \#4060), anti-pPDGFR (Tyr1009, \#3124), and antiphospho-Erk1/2 (Thr202/Tyr204, \#4370).

Human cytokine antibody array (Abcam, Cambridge, USA, ab133998) was performed according to the manufacturer's instruction manual.

For MET activation and neutralization experiments, RKO cells were seeded in 24-well plates at a density of 40,000 cell per well in culture medium containing 10\% FBS. One day before treatment, the wells were refreshed with medium containing $2 \%$ FBS. CAF CM diluted with culture medium (2\% FBS) at 1:2 was added to the cells for $20 \mathrm{~min}$ at $37^{\circ} \mathrm{C}$. Alternatively, the HGF neutralizing antibody was added to the diluted CAF CM and incubated for $1 \mathrm{~h}$ before treatment. The cells were lysed with a RIPA-containing phosphatase inhibitor cocktail for preparation of the WB samples.

\section{Animal Experiments}

Female 6 to 8 week-old age-matched BalbC/nude mice were used for animal studies. The mice were kept in pathogenfree colony cages ( 5 per cage) in an airflow cabinet at $23^{\circ} \mathrm{C}$, $12 / 12 \mathrm{~h}$ day/night cycle, and free access to food and water. The mice were anesthetized with sevoflurane using a small-animal anesthesia system (RWD Life Science, Shenzhen, China). A 68 -mm incision was made near the upper left quadrant of the mice to expose the spleen. CAFs and luciferase-RKO mixed at a ratio of 3:10 PBS were injected to the distal end of the spleen at 130,000 cells in $50 \mu \mathrm{l}$ PBS per mice. Three weeks later, the spleen was surgically removed under anesthesia and the mice were allowed to recover for 3 days. Then, the mice received intraperitoneal injection of luciferin and were subjected to live imaging for visualization of liver metastasis using a system (IVIS Spectrum, Perkin Elmer). Then, the mice were sacrificed and 200 $\mu l$ of luciferin was injected into the portal vein. The liver was then removed and subjected to bioluminescence imaging to confirm the in vivo observation and to rule out peritoneal metastasis caused by leakage of the tumor suspension during inoculation.

\section{Quantitative Reverse-Transcription PCR (RT-qPCR)}

Cells were lysed in TRIzol (Invitrogen), and total RNA was extracted according to the instruction manual. cDNA was obtained using Hifair ${ }^{\circledR}$ II 1st-Strand cDNA Synthesis SuperMix for qPCR (Yeason, Shanghai, China). Real-time PCR was performed using the 7500 Fast Real-Time PCR System (Applied Biosystems, Foster City, CA, USA) with Hieff $^{\circledR}$ qPCR SYBR
Green Master Mix (Low Rox Plus) (Yeason, Shanghai, China) in a two-step reaction. Analysis of the relative expression of target gene was performed by the comparative Ct value.

\section{Cell Proliferation Assay}

CCK-8 assay was used to assess cell proliferation. Cells were seeded in 96-well plates at 3,000 cells per well in triplicates. After culturing under the designated conditions, the supernatant was replaced with $100 \mu \mathrm{l}$ fresh culture medium containing $10 \%$ FBS and $10 \mu \mathrm{l}$ CCK- 8 reagent and incubated for 1.5 before measuring with a microplate reader at $450 \mathrm{~nm}$. A blank well containing only culture medium and CCK- 8 reagent was also measured as a background signal, which was subtracted from the experiment readouts.

\section{Statistical Analysis}

All analysis was performed in GraphPad Prism 8. Results were presented as means \pm SD. Statistical analysis was performed using one-way ANOVA with Bonferroni post hoc test or the Student's $t$-test. Survival analysis was performed using logrank (Mantel-Cox) test. $P<0.05$ was considered to be statistically significant.

\section{RESULTS}

\section{The Localization and Expression of RAB31 in CRC}

Since RAB31 has never been studied in colon cancer, we validated a commercially available RAB31 antibody using WB and immunocytochemistry (Supplementary Figure 1) and explored the expression and distribution of RAB31 in paraffin-fixed tissue from colon cancer patients. Immunofluorescence staining of normal mucosa and paired tumor tissue showed that RAB31 was highly expressed in goblet cells with dispersed clusterlike distribution in the cytoplasm. The stromal compartment of normal tissue did not show significant staining of RAB31 compared to the goblet cells. In tumor tissue, RAB31 expression in the cancer cells were generally low, while high expression was detected in the tumor stroma in a subgroup of samples. Partial colocalization with $\alpha$-SMA, a marker for CAFs (Figure 1A), or vimentin, a marker for stromal cells (Supplementary Figure 2), was observed, suggesting a functional role for RAB31 in CAFs. RAB31 was detected by WB in 3 out of 10 CRC cell lines, and in 2 primary CAFs and paired normal fibroblasts (Figure 1B). As RAB family proteins are generally membrane associated, their functions may be reflected by their subcellular localization. We transiently expressed a RAB31-EGFP fusion protein in primary CAFs and found that RAB31 was accumulated near the cell nucleus and colocalized strongly with the Golgi complex marker TGN46. In the cytoplasma, RAB31 was distributed as tiny clusters resembling intracellular vesicles (Figure 1C), suggesting that RAB31 may be a mediator of Golgi-endosome transportation, which is consistent with early studies $(6,7)$.

Next, we analyzed the expression of RAB31 in colorectal tumor samples based on the consensus molecular subtypes (CMS) classification. Normalized gene expression data (25) of 2,844 patients were sorted by RAB31 expression level and 

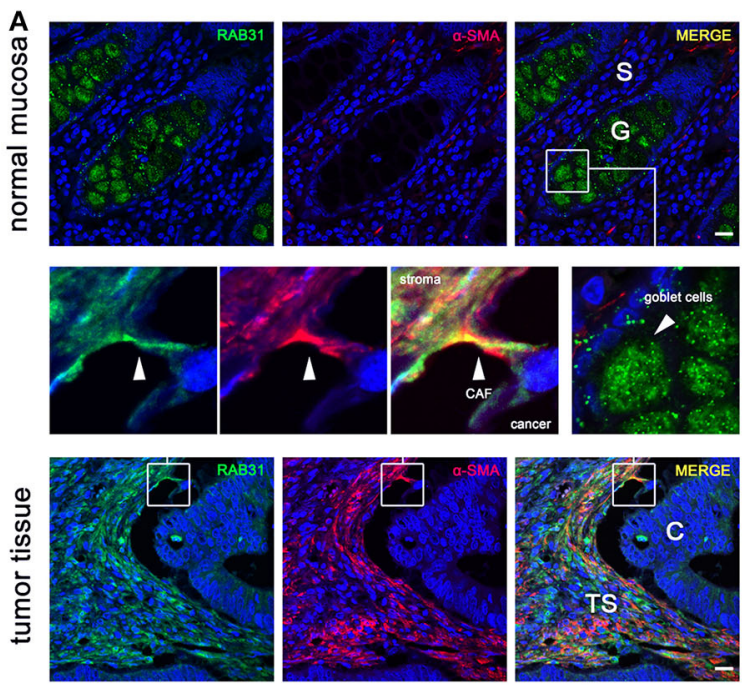

C
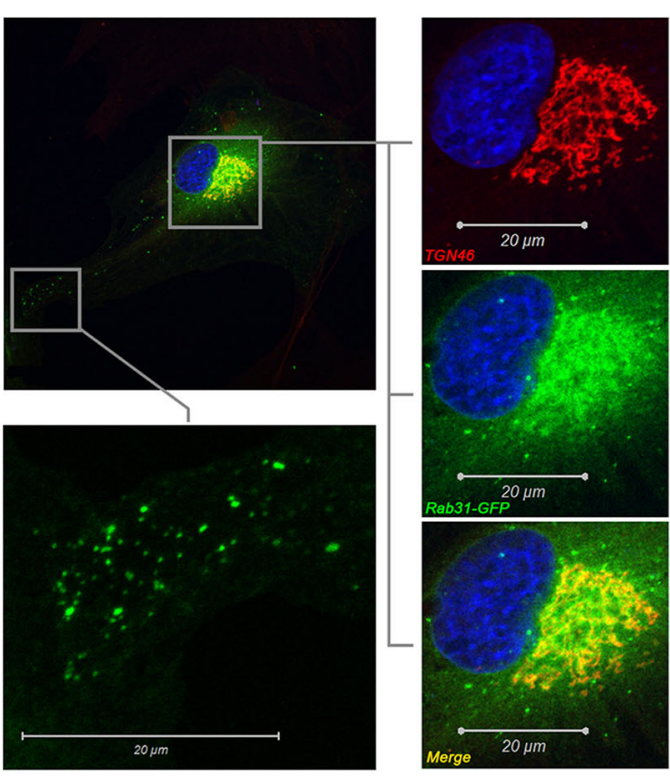

B

B

RAB31

GAPDH
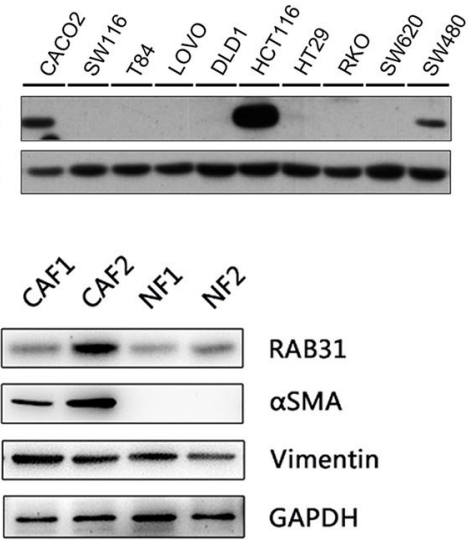

\section{D}
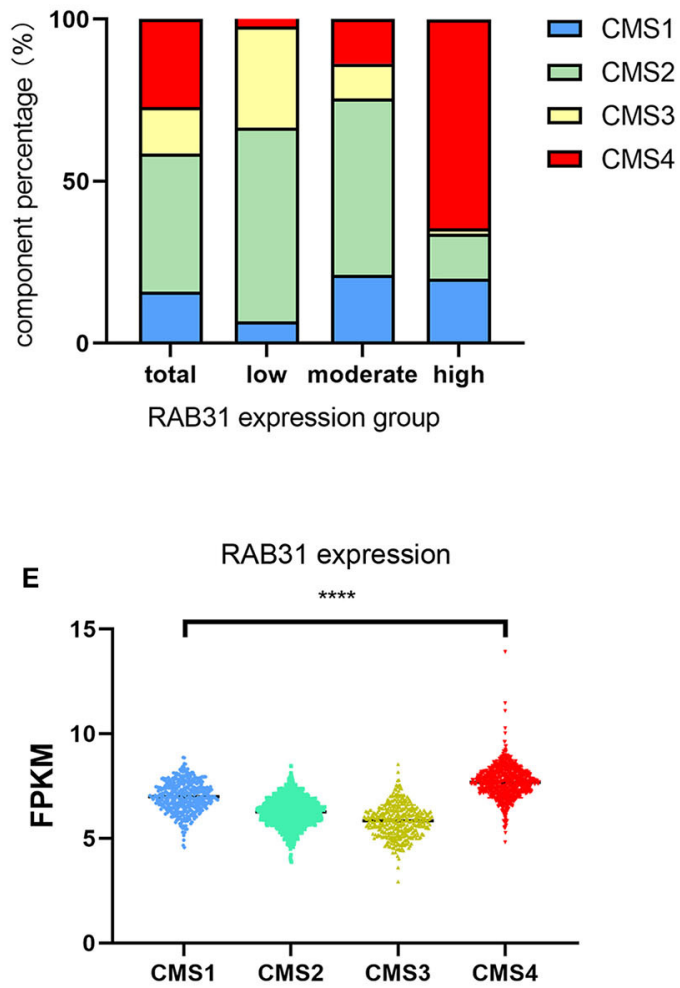

FIGURE 1 | The localization and expression of RAB31 in colon cancer. (A) Paired colon cancer tissue and adjacent normal mucosa co-stained for RAB31 (green) and the CAF marker $\alpha$-SMA (red) show significant staining of RAB31 in the goblet cells of normal mucosa which are balloon-like cells clustered together and in the tumor stroma where it partially colocalizes with $\alpha$-SMA. Scale bar $=50 \mu \mathrm{m}$. S, normal stroma; C, cancer cells; TS, tumor stroma; G, goblet cells. (B) WB detects the expression of RAB31 in 3 out of 10 CRC cell lines and in paired primary CAFs and NF. (C) Subcellular localization of RAB31-EGFP shows colocalization with the trans-Golgi network marker TGN46 and cluster-like distribution in the cytoplasm of primary CAFs. (D) The CMS component of CRC patients in RAB31 high ( $n=941$ ), moderate $(n=941)$, and low expression $(n=962)$ groups with a cut-off value of 6.22 and 7.14 FPKM. The RAB31 high expression group holds the largest portion of CMS4 CRC patients. (E) RAB31 expression is highest in CMS4 (stromal type) colorectal cancer and significantly higher than CMS1 (immune type). Data shown as mean \pm s.d. ${ }^{* \star * *} P<0.0001$; statistical significance was determined by one-way ANOVA with Bonferroni post hoc test.

equally divided into RAB31 high, medium, and low expression groups. The RAB31 high group consisted of a significantly higher proportion of CMS4 (high stromal content) CRC samples compared to the overall CMS4 proportion $(64.55 \%$ vs. 27.10\%) (Figure 1D). In addition, RAB31 overall expression was highest in the CMS4 group, significantly higher than that in the CMS1 (immune related) group, which had the second highest expression of RAB31 in the 4 CMS groups 
(CMS4 vs. CMS1; $7.69 \pm 0.75$ vs. $6.99 \pm 0.70 ; P<0.0001$ ) (Figure 1E).

These results suggest that RAB31 may play a functional role in cellular components of the tumor stroma such as CAFs.

\section{Stromal Expression of RAB31 Is Associated With Poor Prognosis in CRC}

To further investigate the clinical significance of RAB31 expression in colon cancer, we performed immunohistological staining of RAB31 in 98 CRC tumor samples (Table 1). The

TABLE 1 | Basic characteristics of CRC patients.

\begin{tabular}{llc}
\hline Mean age (SD) & & $68.9 \pm 11.8$ \\
Sex (\%) & Male & $52(53.1 \%)$ \\
TNM stage & Female & $46(46.9 \%)$ \\
& I & \\
II & $6(6.1 \%)$ \\
III & $53(54.1 \%)$ \\
IV & $35(35.7 \%)$ \\
& & $4(4.1 \%)$
\end{tabular}

Site

$\begin{array}{lc}\text { Right colon } & 49 \\ \text { Left colon } & 48 \\ \text { N/A } & 1\end{array}$

expression of RAB31 was graded in both the cancer cells and tumor stromal compartment using a 3-tier scale: 1, no staining or weak staining; 2, moderate staining; 3, strong staining. The results were then divided into negative staining for grade 1; positive staining for grade 2 and 3 . While RAB31 may be expressed in both cancer cells and stromal cells (Figures 2A-D), a higher percentage of stromal RAB31 expression was observed (56.12\% vs. 35.71\%) (Figure 2F). Kaplan-Meier survival analysis was performed on RAB31positive vs. negative groups in cancer cells and stromal compartments, respectively. We found that CRC with stromal expression of RAB31 was associated with poor survival in comparison to those with negative staining. Interestingly, expression of RAB31 in cancer cells was not correlated with survival (Figure 2E).

\section{CAF Expressed RAB31 Promotes CRC Cell Migration and Metastasis}

To determine whether elevated expression of RAB31 in CAFs can affect the biological properties of CRC cells, we overexpressed RAB31 in two primary colon cancer-derived CAFs using lentiviral infection. The proliferation rates of the CAFs were unaltered following RAB31 overexpression (Figure 3A). CM obtained from these CAFs had no observable effect on cancer cell proliferation (Figure 3B) but stimulated the transwell migration of RKO and LOVO cells significantly in comparison to vector control $(P<0.0001)$ (Figure 3C), suggesting an increase in

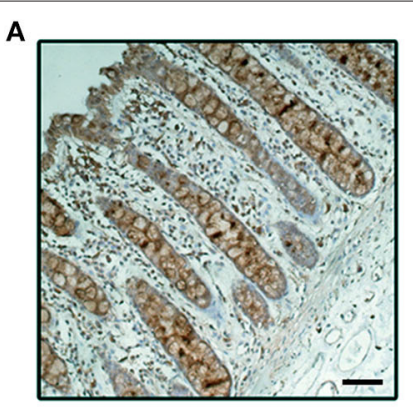

C
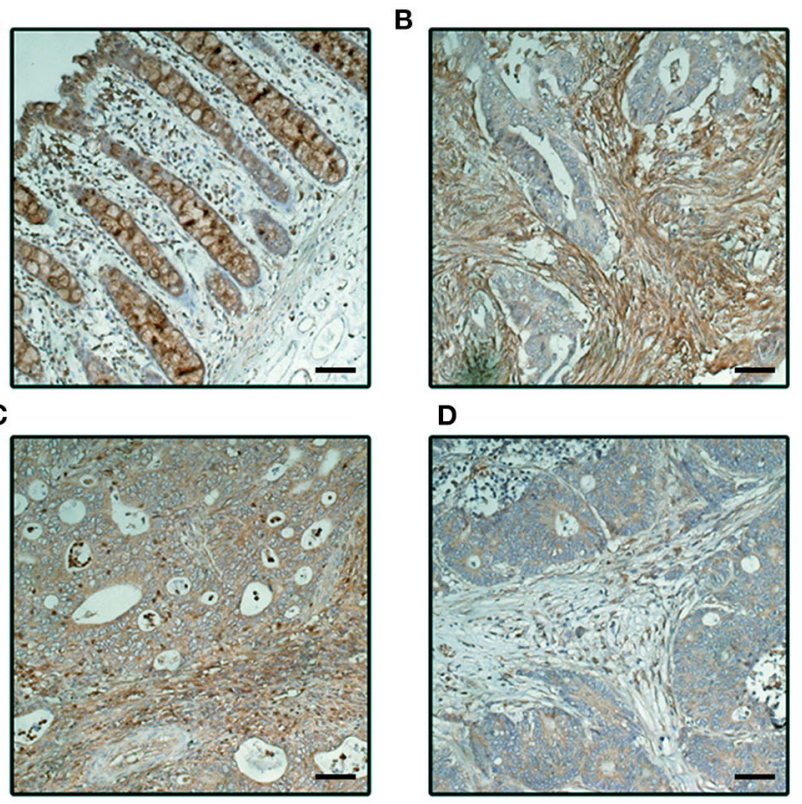

D

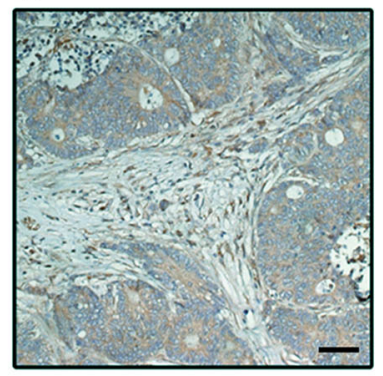

E

\begin{tabular}{l|cc}
\hline & Ca RAB31 + & Ca RAB31 - \\
\hline TS RAB31 + & $12 / 98$ & $43 / 98$ \\
TS RAB31 - & $23 / 98$ & $20 / 98$ \\
\hline
\end{tabular}

F

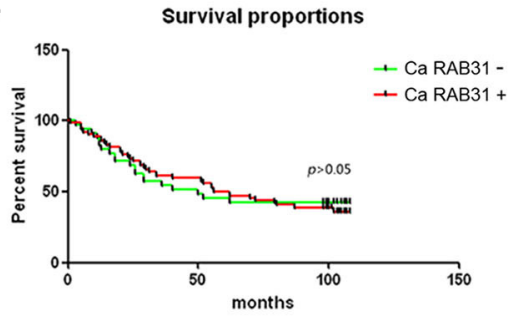

Survival proportions

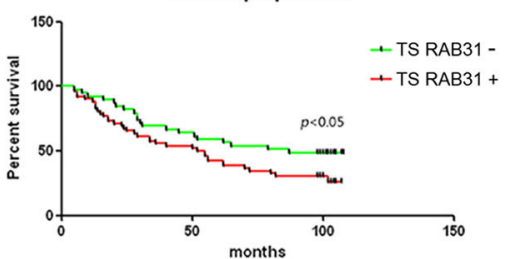

FIGURE 2 | Stromal expression of RAB31 is associated with poor prognosis in CRC. RAB31 was stained in 98 colon cancer tissue and paired normal mucosa. RAB31 was found in goblet cells in normal tissue (A) and may be richly expressed in stroma or cancer cells (B,C), or nearly absent in both compartments in some samples (D). Scale bar $=50 \mu \mathrm{m}$. (E) RAB31 was evaluated in the cancer cells and stromal compartments for each sample. Summarized data of IHC results shows the fraction of RAB31-positive/negative samples out of 98 patients in terms of tumor stroma or in cancer cell expression. TS RAB31: tumor stromal RAB31 expression, Ca RAB31: cancer cell RAB31 expression. (F) Kaplan-Meier survival analysis of RAB31 expression based on stromal or cancer cell expression suggests that only stromal RAB31 expression was significantly associated with survival, $P=0.018$, by log-rank (Mantel-Cox) test. 

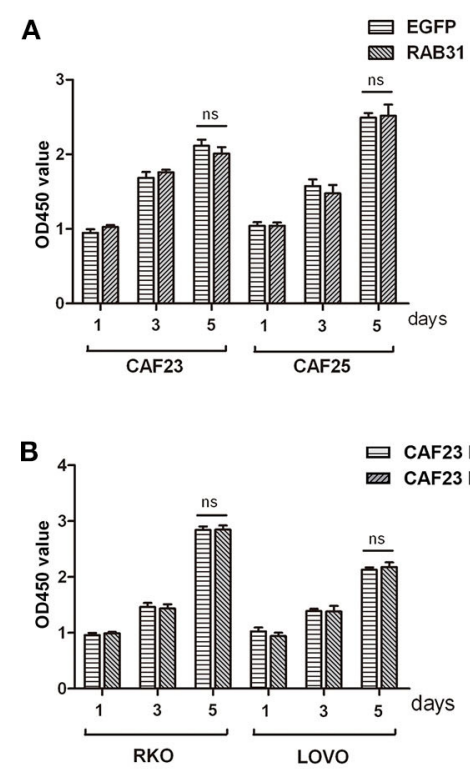
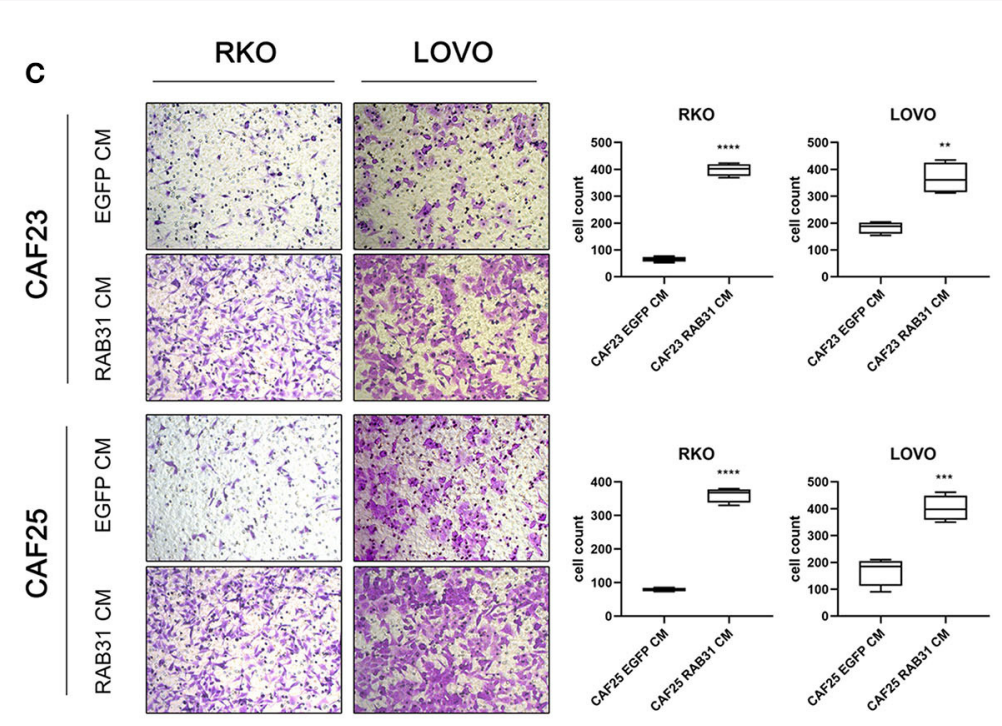
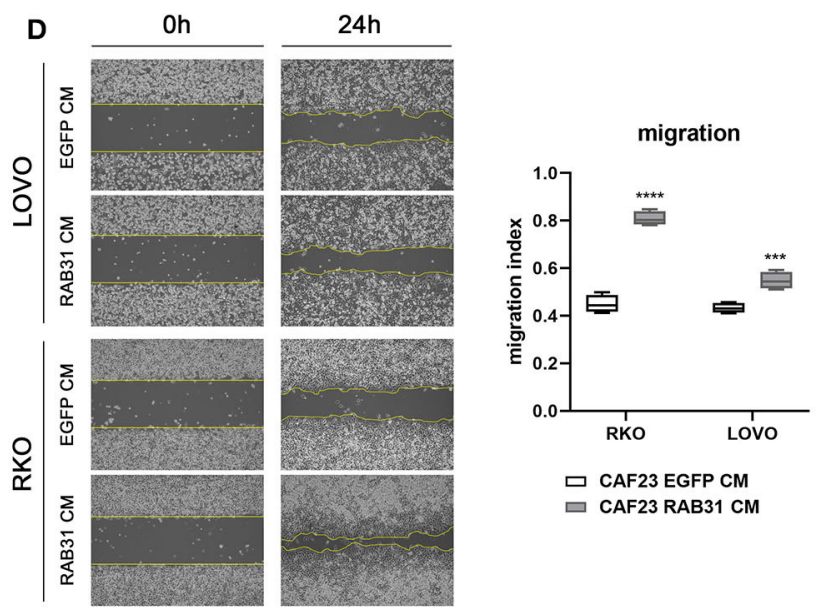

E

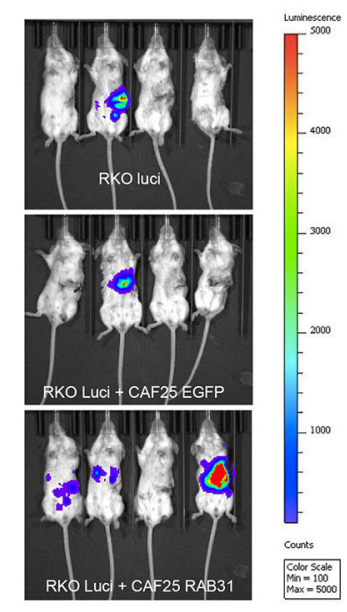

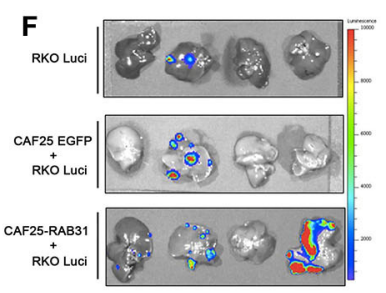

G

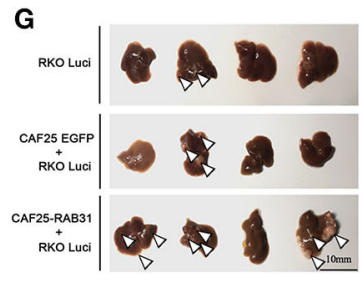

FIGURE 3 | CAF-expressed RAB31 promote colorectal cancer cell migration and metastasis. (A) The proliferation rate of CAF23 and CAF25 overexpressing RAB31 was unchanged compared to vector control CAFs measured by cck-8 assay. (B) Proliferation of RKO and LOVO cells incubated with conditioned medium obtained from RAB31-overexpressing CAFs or vector control CAFs measured by CCK-8 assay. (C) Conditioned medium of CAFs overexpressing RAB31 significantly facilitated the migration of CRC cell lines RKO and LOVO compared to vector control measured by Transwell assay at $45 \mathrm{~h}$. (D) Wound healing assay of RKO and LOVO treated with CM from RAB31-overexpressing CAFs or vector control CAFs for $24 \mathrm{~h}$. (E) RKO colon cancer cells expressing luciferase inoculated into the distant end of the spleen in mixture with RAB31-overexpressed CAFs exhibited increased liver metastasis compared to that mixed with or without WT CAFs. Images of livers with metastatic lesions at 30 days detected by live imaging. (F) Bioluminescence image of metastatic lesions in isolated livers and (G) by direct observation, indicated by white arrowheads. (A-D) Data shown as mean \pm s.d. ${ }^{\star \star \star \star} P<0.0001,{ }^{\star \star \star} P<0.001,{ }^{\star \star} P<0.01$; statistical significance was determined by Student's $t$-test.

CAF-secreted migration-promoting factors. This phenomenon was further supported by wound healing assay (Figure 3D). Interestingly, while the fibroblast CM induced morphological changes in certain cell lines, the proliferation of RKO and LOVO was unaltered. The percentage of FBS supplement or the addition of fibroblast growth factor seemed to have the most prominent effect in facilitating RKO and LOVO proliferation (Supplementary Figure 3).

To further confirm our observation in vivo, we used a murine xenograft model for CRC liver metastasis by spleen injection of luciferase-expressing RKO. Results showed that mixed inoculation of RAB31-overexpressing CAFs and RKO exhibited a higher liver metastasis rate compared to the $\mathrm{RKO} /$ control CAF group or RKO alone (75, 41.67, and 25\%, respectively) (Figure 3E, Table 2 and Supplementary Figure 4). After live imaging, the livers were excised for organ luminescence detection and direct observation of metastatic lesions, which was consistent with the live imaging results (Figures 3F,G).

These results suggest that CAF-expressed RAB31 may promote the migration of CRC cells through paracrine actions. Moreover, our murine xenograft model supports the clinical 
observation that higher RAB31 expression in the stroma is associated with CRC liver metastasis (Stage IV).

\section{CAF Expressed RAB31 Promotes CRC Cell Migration Through Regulating HGF Secretion}

To further identify the growth factors or cytokines responsible for the enhanced migration-inducing ability of CM from RAB31overexpressing CAFs, we used a cytokine antibody array panel to search for differentially secreted factors. Quantification of the immune blots revealed TGF- $\beta$, HGF, EGF, and IGFBP-2

TABLE 2 | Mice liver metastasis model.

\begin{tabular}{|c|c|c|c|}
\hline Group & Total no. of mice & Liver metastasis & Rate (\%) \\
\hline RKO-luciferase & 8 & 2 & 25.00 \\
\hline RKO-luciferase+CAF25 EGFP & 12 & 5 & 41.67 \\
\hline RKO-luciferase+CAF25 RAB31 & 12 & 9 & 75.00 \\
\hline
\end{tabular}

and TIM3 (Figure 4A) to be significantly elevated in the $\mathrm{CM}$ of RAB31-overexpressing CAFs. Expression changes of other secreted factors can be found in Supplementary Figure 7. CAFs have been known to secrete a myriad of cytokines and growth factors such as SDF-1, IL-6, IL-8, TGF- $\beta$, and $\operatorname{HGF}(31,32)$. While many studies claim that these CAFsecreted factors may enhance tumor cell migration and induce epithelial-mesenchymal transition (EMT), they may play distinct roles in different types of cancers. Transwell assay results showed that CAF-derived CM potently promoted RKO migration. In addition, among 7 well-studied CAFsecreted factors, HGF exhibited the strongest effect in promoting RKO migration $(P<0.0001)$ (Figures 4B,C). The combined results of cytokine array and transwell assay suggest that HGF may be a key factor in CAF-mediated migration of colon cancer cells.

Next, we treated RKO cells with CM from control or RAB31overexpressing CAFs and examined the activity of the HGF/Met signaling pathway. Results showed that incubation with control CAF-derived CM increased MET phosphorylation in RKO; this effect was further enhanced when incubated with that from

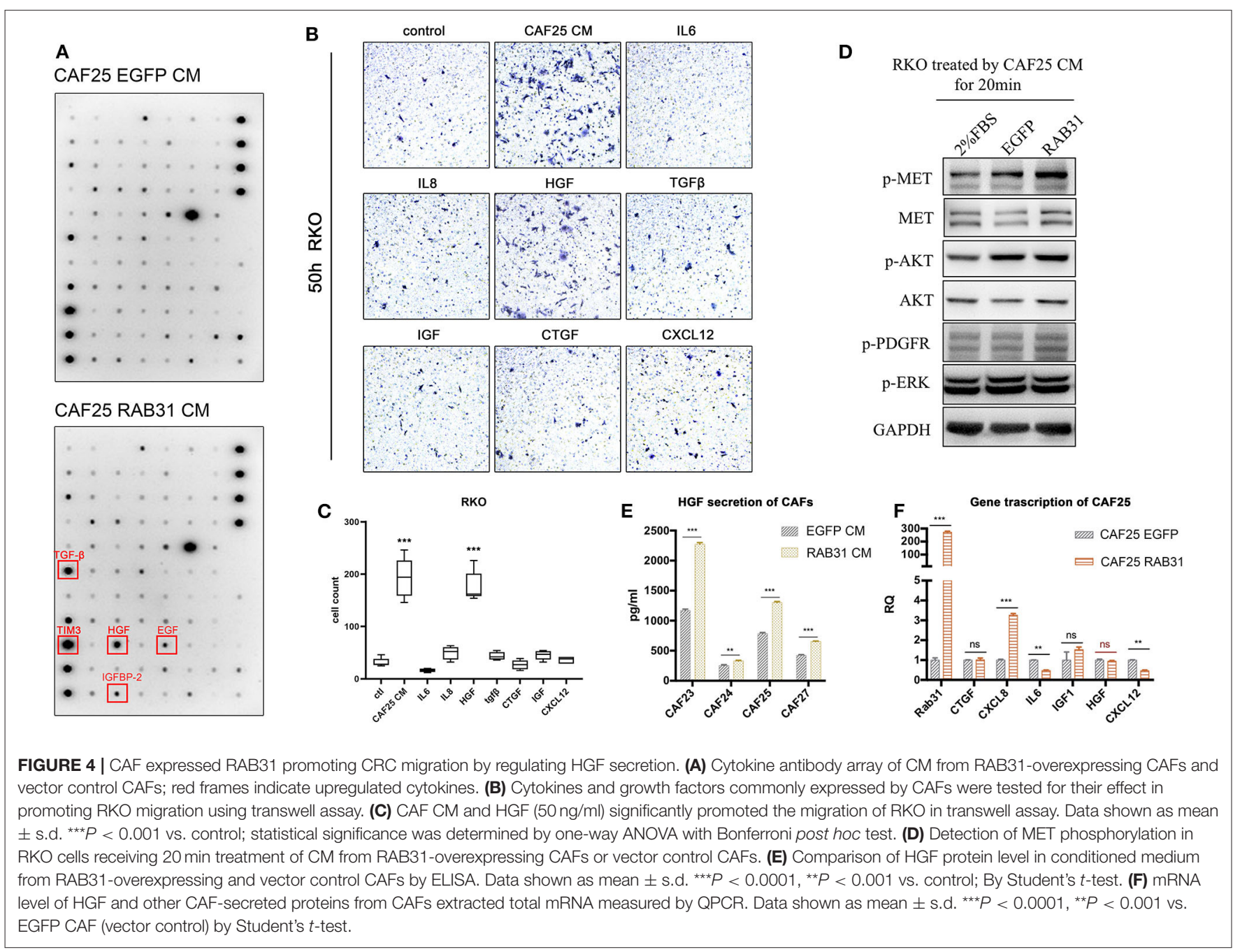



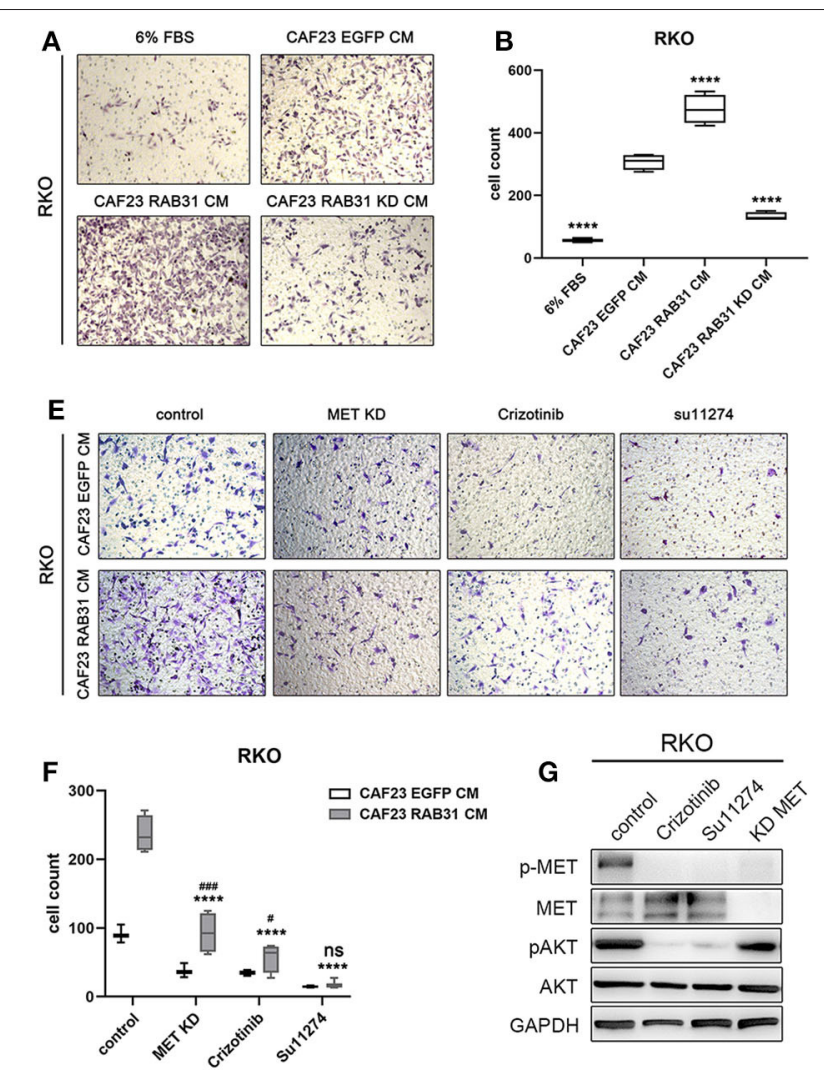
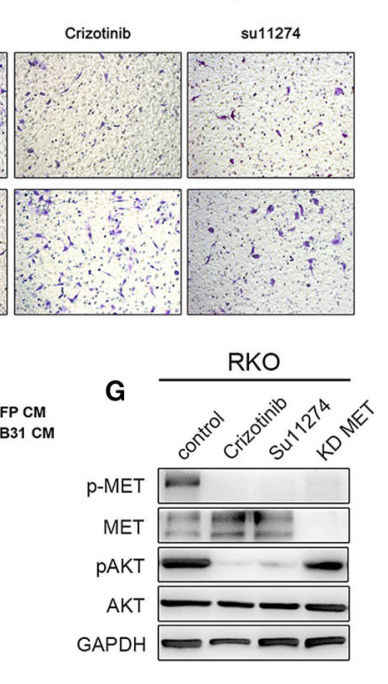
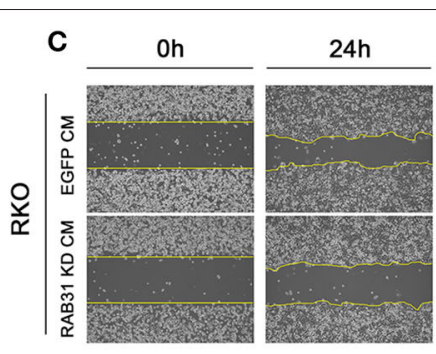

D
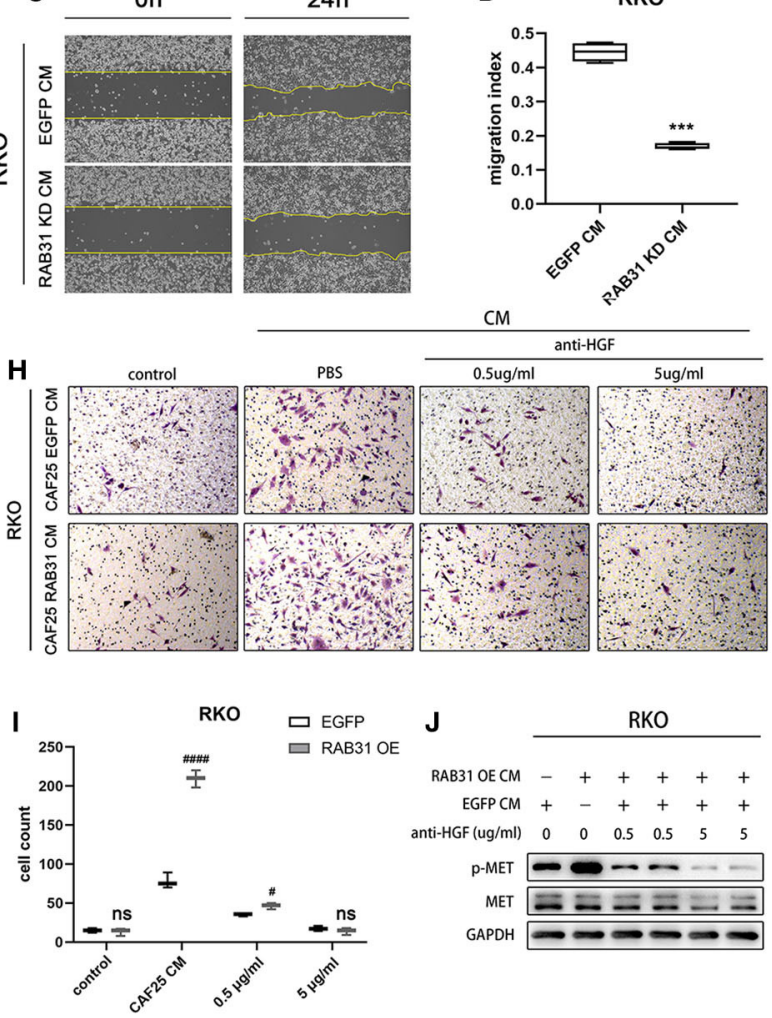

FIGURE 5 | HGF/MET signaling blockade abolishes colon cancer cell migration mediated by RAB31 in CAFs. (A) RKO migration measured by Transwell assay at $50 \mathrm{~h}$. The lower chamber contained primary culture medium supplemented with $6 \%$ FBS for control or CAF derived CM. (B) The migration of RKO was significantly enhanced by vector control (EGFP) CAF CM, and further enhanced by RAB31 overexpressing CAF CM measured by Tranwell assay. Knockdown of RAB31 in CAF by lentivirus significantly reduced the migration of RKO. ${ }^{\star \star \star \star} P<0.0001$ vs. CAF23 EGFP CM. (C,D) Wound healing assay of RKO cells incubated with CM from vector control CAFs or RAB31 knockdown CAFs. ${ }^{\star \star \star} P<0.001$ vs. RAB31 KD CM. (E) Migration of RKO cells treated with RAB31 overexpressing CAF CM or EGFP CAF CM (vector control) in the presence of MET inhibitors Crizotinib (100 nM) or Su11274 $(5 \mu \mathrm{M})$ measured by Transwell assay at $45 \mathrm{~h}$. (F) Blockade of MET signaling significantly inhibited the CAF CM mediated RKO migration and ameliorated the promigratory effect of RAB31 overexpression. ${ }^{* \star *} P<0.0001$ vs. CAF23 RAB31 CM control; \#\#\#P<0.001, \#P < 0.05 vs. CAF23 EGFP CM. (G) WB indicated that treatment of RKO with CAF23 EGFP CM increased the phosphorylation of MET, which was blocked by MET inhibitors or by MET knockdown in RKO. (H,I) Neutralizing HGF in CAF25 EGFP CM and CAF25 RAB31 CM inhibited migration of RKO cells by Transwell assay at $45 \mathrm{~h}$ in a dose dependant manner and abolished the difference of the two CMs at $5 \mu \mathrm{g} / \mathrm{ml}$. \#\#\#\# $P<0.0001$, \# $P<0.05 \mathrm{vs}$. CAF23 EGFP CM. (J) WB results showed that the increased MET phosphorylation in RKO induced by CM from RAB31 overexpressing CAFs was diminished upon preincubation with anti-HGF antibody at $5 \mu \mathrm{g} / \mathrm{ml}$. (D,F,G,I) Data shown as mean \pm s.d. Statistical significance was determined by Student's $t$-test.

RAB31-overexpressing CAFs (Figure 4D). HGF protein level was significantly increased in the CM of four different primary RAB31-overexpressing CAFs compared to their vector control counterparts by ELISA detection (Figure 4E), while the mRNA level of HGF was unchanged (Figure 4F).

These results suggest that RAB31 may mediate posttranslational regulation of $\mathrm{HGF}$ expression in CAFs, which subsequently increase HGF/MET signaling in cancer cells.

\section{HGF/Met Signaling Blockade Inhibited CRC Cell Migration Mediated by RAB31 Expression in CAFs}

Finally, we knocked down RAB31 in CAF23 significantly using lentiviral infection (Supplementary Figure 5) and found that the increase in RKO migration induced by CAF $\mathrm{CM}$ was partially abolished as measured by transwell assay
(Figures 5A,B). In addition, wound healing assay also showed that the downregulation of RAB31 hampered CAF CM induced migration (Figures 5C,D). To further demonstrate that the HGF/Met pathway was involved, we compared the effect of CM from RAB31-overexpressing CAFs and vector control CAFs on RKO migration in the presence of the small molecule MET inhibitors crizotinib or Su11274, or by knockdown of MET in RKO. Results showed that knockdown of MET in RKO or treatment with crizotinib $(100 \mathrm{nM})$ or Su11274 $(5 \mu \mathrm{M})$, respectively significantly reduced the migration of RKO induced by CAF-derived CM. While RAB31-overexpressing CAF-derived $\mathrm{CM}$ increased RKO migration to a greater extent compared to control CAFs, this difference was diminished in the MET knockdown and crizotinib groups and completely abolished in the Su11274 group (Figures 5E,F). The inhibitory effects of the two inhibitors and MET knockdown were validated by WB which showed significantly decreased phosphorylation of MET 
and downstream Akt signaling upon inhibitor preincubation (Figure 5G). Because there are potentially other growth factors that can activate MET such as IGF (33), we further complemented our study by neutralizing HGF in CAF CM. The HGF-neutralizing antibody reaches a maximum effect at $5 \mu \mathrm{g} / \mathrm{ml}$ as indicated by significantly decreased p-MET (Supplementary Figure 8). Transwell assay showed that neutralizing HGF inhibited migration of RKO and abolished the pro-migratory difference between CAF25 RAB31 CM and CAF25 EGFP CM (Figures 5H,I). In addition, $5 \mu \mathrm{g} / \mathrm{ml}$ neutralizing antibody reduced CM-induced p-MET of the two CAFs to similar levels (Figure 5J), suggesting HGF as the primary growth factor responsible for CM induce MET activation.

Taken together, these results strongly suggest that RAB31 overexpression in CAFs may promote RKO migration through paracrine regulation of HGF/MET signaling.

\section{DISCUSSION}

In our previous study, RAB31 expression was found to be significantly elevated in stage IV CRC compared to normal tissue and stage I-III CRC (2). In this study, RAB31 was further identified to be highly expressed in CMS4 or stromal type CRC. Stromal RAB31 expression had worse prognosis in colon cancer. We demonstrate that increased RAB31 expression in CAFs, a major component of stromal cells, may promote colon cancer progression by increasing HGF secretion which subsequently activates HGF/Met signaling in CRC cells.

While studies have investigated the role of RAB31 in the cancer cells of hepatic cancer, breast cancer, pancreatic cancer, gastric cancer, and glioblastoma (10-12, 34, 35), we described the functional role of RAB31 in colon cancer-derived CAFs because its expression in the tumor stroma but not cancer cells predicted poor survival. RAB31 was expressed in goblet cells of normal colon mucosa as well as in cancer cells in a fraction of colon cancer samples. Its expression in cancer cells may be a premature feature of goblet cells since cancer cells are genetically unstable and incompletely differentiated (36). On the other hand, CAFs are genetically stable but heterogeneous in origin $(37,38)$. Functional CAF subpopulations have been classified based on expression of specific marker proteins $(39,40)$. Our results show that not all CAFs express RAB31 in paraffinfixed tissue by IHC. In addition, WB results showed that cultured CAFs did not show a significantly higher expression of RAB31 compared to their NF counterpart. Therefore, increased RAB31 expression in the tumor stromal may be induced by specific factors in the microenvironment or may mark a specific subtype of CAFs. This requires further investigation under the basis of an authentic subgroup classification system of CAFs, which has yet to be established.

CAFs secrete a myriad of proteins such as SDF1 (CXCL12), TGF- $\beta$, IGF, and CTGF, which have been shown to induce EMT (40-43), increasing the motility of cancer cells to promote metastasis. Using cytokine antibody array, we found several cytokines and growth factors upregulated in the CM of RAB31overexpressing CAFs. Among them, HGF seemed to have the most pronounced effect in promoting the migration of CRC cell lines RKO and LOVO. HGF is a fibroblast-derived growth factor found to stimulate the mobility of epithelial cells (4446) and was later shown to play an important role in the progression of malignant tumors (26). HGF is the canonical ligand for the receptor tyrosine kinase MET. However, it has been demonstrated that MET can be activated through HGFindependent mechanisms such as gene amplification, mutation, and transcriptional upregulation (47-49). Moreover, interaction with other proteins such as Integrin $\alpha 6 \beta 4$, intercellular adhesion molecule 1 (ICAM-1), and CD44v6 enhances MET activation $(50,51)$. Transactivation of MET by GPCRs and EGFR which bind to multiple ligands has also been reported (52). In our study, we found that treatment with CAF-derived CM promoted MET activation, which was further enhanced using CM from RAB31-overexpressing CAFs. Moreover, depletion of HGF using a polyclonal antibody reduced RKO MET activation by CM from RAB31-overexpressing CAFs and control CAFs to similar levels, suggesting that enhanced MET activation by RAB31overexpressing CAF CM was indeed mediated primarily by HGF and not by other soluble factors.

Approximately $70 \mathrm{RAB}$ proteins have been identified in the human genome. The expression of RAB family proteins is dysregulated in a variety of tumor types, which may alter the biological properties of cancer cells such as proliferation, migration, and invasion (9). RAB proteins switch between a GTP bound "on state" and GDP bound "off state," allowing them to bind effector proteins and determining localization on membrane compartments or the cytoplasm (53). RABs that reside in the Golgi complex are implicated in maintaining the Golgi structure, Golgi to endosome trafficking, and protein sorting. Although we described the subcellular distribution of RAB31 as vesicle-like clusters, we were not able to verify whether these were anterogradetransporting vesicles. The RAB31 clusters did not colocalize with early endosomes or lysosomes (Supplementary Figure 6), which exclude its role in the endosome/lysosome degradation pathway and, to some extent, suggest that it may participate in anterograde trafficking or secretion of certain proteins.

RAB31 overexpression in CAFs resulted in increased HGF protein levels in the conditioned medium while the mRNA levels were unchanged. This further indicates that the regulation of HGF occurs at the posttranslational level. Other RAB family proteins such as $\mathrm{RAB} 3 \mathrm{C}$ have been reported to regulate the secretion of IL6 to promote colorectal cancer metastasis, the underlying mechanism of which still needs to be elucidated (54). Different RABs may play distinct roles in the secretary pathway. The Golgi RAB8 and RAB26 interact with adrenergic receptors through different binding domains, respectively, to regulate the proper membrane expression of these GPCRs (55). RAB27 directly interacts with myosinVa within a functional complex to facilitate the transport of dense core secretory granules to the plasma membrane in hormone and neuropeptide-producing cells or mast cells (56, 57). RAB31 has been reported to directly interact with the adapter protein APPL2, which is required for FcR-mediated 
PI3K/Akt signaling in macrophages (58). RAB31 has also been shown to co-immunoprecipitate with the p75 neurotrophin receptor (59) and with EGFR (60), playing an important role in the trafficking of the respective receptors. The specific binding domain or motif responsible for interaction with other proteins has never been identified in RAB31. Nevertheless, it is still possible that RAB31 may bind to HGF directly to facilitate its anterograde trafficking and secretion or serve as a functional component of a transportation complex that promotes HGF secretion.

Our study mainly focused on RAB31 in CAFs; however, the tumor stroma consists of a variety of cells including macrophages, lymphocytes, and epithelial cells. It is possible that RAB31 expressed in other cell populations may also affect the biological properties of CRC tumor cells. Therefore, it would be interesting to investigate the functional role of RAB31 in other cell types such as immune cells since CMS1 (immune infiltration type) colorectal cancer also express high levels of RAB31.

In conclusion, we present RAB31 as an important regulator of HGF secretion in CRC-derived cancer-associated fibroblasts, which plays an important role in the progression of colon cancer through activating HGF/Met signaling in cancer cells.

\section{DATA AVAILABILITY STATEMENT}

The raw data supporting the conclusions of this article will be made available by the authors, without undue reservation.

\section{REFERENCES}

1. Harrison S, Benziger $H$. The molecular biology of colorectal carcinoma and its implications: a review. Surgeon. (2011) 9:200-10. doi: 10.1016/j.surge.2011.01.011

2. Yu J, Li X, Zhong C, Li D, Zhai X, Hu W, et al. High-throughput proteomics integrated with gene microarray for discovery of colorectal cancer potential biomarkers. Oncotarget. (2016) 7:75279-92. doi: 10.18632/oncotarget.12143

3. Stenmark H. Rab GTPases as coordinators of vesicle traffic. Nat Rev Mol Cell Biol. (2009) 10:513-25. doi: 10.1038/nrm2728

4. Kelly EE, Horgan CP, Goud B, Mccaffrey MW. The Rab family of proteins: 25 years on. Biochem Soc Trans. (2012) 40:1337-47. doi: 10.1042/BST20120203

5. Goody RS, Muller MP, Wu YW. Mechanisms of action of Rab proteins, key regulators of intracellular vesicular transport. Biol Chem. (2017) 398:565-75. doi: $10.1515 / \mathrm{hsz}-2016-0274$

6. Rodriguez-Gabin AG, Cammer M, Almazan G, Charron M, Larocca JN. Role of rRAB22b, an oligodendrocyte protein, in regulation of transport of vesicles from trans Golgi to endocytic compartments. J Neurosci Res. (2001) 66:1149-60. doi: 10.1002/jnr.1253

7. Rodriguez-Gabin AG, Yin X, Si Q, Larocca JN. Transport of mannose-6phosphate receptors from the trans-Golgi network to endosomes requires Rab31. Exp Cell Res. (2009) 315:2215-30. doi: 10.1016/j.yexcr.2009.03.020

8. Rodriguez-Gabin AG, Ortiz E, Demoliner K, Si Q, Almazan G, Larocca JN. Interaction of Rab31 and OCRL-1 in oligodendrocytes: its role in transport of mannose 6-phosphate receptors. J Neurosci Res. (2010) 88:589604. doi: $10.1002 /$ jnr.22236

9. Tzeng H, Wang Y. Rab-mediated vesicle trafficking in cancer. J Biomed Sci. (2016) 23:70. doi: 10.1186/s12929-016-0287-7

\section{ETHICS STATEMENT}

This project was approved by the Ethical Committee of the Second Affiliated Hospital of Zhejiang University School of Medicine and written informed consent was obtained from all patients.

\section{AUTHOR CONTRIBUTIONS}

TY and WZ contributed equally to this study, performed the experiments, analyzed the data, and wrote the manuscript. WZ designed the experiments. TY, XQ, and LY funded the project. $\mathrm{ZS}$ and DK checked and revised the manuscript and confirmed all the data in the manuscript. GX and WJ performed the experiments for the revised manuscript. All authors read and approved the final manuscript.

\section{FUNDING}

This work was supported by the National Natural Science Foundation of China (nos. 81902959, 81902818, and 81702331) and Zhejiang Provincial Natural Science Foundation of China (nos. LQ18H160014 and LQ19H160037).

\section{SUPPLEMENTARY MATERIAL}

The Supplementary Material for this article can be found online at: https://www.frontiersin.org/articles/10.3389/fonc. 2020.01747/full\#supplementary-material
10. Grismayer B, Solch S, Seubert B, Kirchner T, Schafer S, Baretton G, et al. Rab31 expression levels modulate tumor-relevant characteristics of breast cancer cells. Mol Cancer. (2012) 11:62. doi: 10.1186/1476-4598-11-62

11. Sui Y, Zheng X, Zhao D. Rab31 promoted hepatocellular carcinoma. (HCC) progression via inhibition of cell apoptosis induced by PI3K/AKT/Bcl-2/BAX pathway. Tumour Biol. (2015) 36:8661-70. doi: 10.1007/s13277-015-3626-5

12. Tang CT, Liang Q, Yang L, Lin XL, Wu S, Chen Y, et al. RAB31 targeted by MiR-30c-2-3p regulates the GLI1 signaling pathway, affecting gastric cancer cell proliferation and apoptosis. Front Oncol. (2018) 8:554. doi: 10.3389/fonc.2018.00554

13. Kotzsch M, Sieuwerts AM, Grosser M, Meye A, Fuessel S, MeijerVan Gelder ME, et al. Urokinase receptor splice variant uPAR-del4/5associated gene expression in breast cancer: identification of rab31 as an independent prognostic factor. Breast Cancer Res Treat. (2008) 111:229-40. doi: 10.1007/s10549-007-9782-6

14. Choi J, Gyamfi J, Jang H, Koo JS. The role of tumor-associated macrophage in breast cancer biology. Histol Histopathol. (2018) 33:133-45. doi: 10.14670/HH-11-916

15. Najafi M, Hashemi Goradel N, Farhood B, Salehi E, Nashtaei MS, Khanlarkhani N, et al. Macrophage polarity in cancer: a review. J Cell Biochem. (2019) 120:2756-65. doi: 10.1002/jcb.27646

16. Rahmatizadeh F, Gholizadeh-Ghaleh Aziz S, Khodadadi K, Lale Ataei M, Ebrahimie E, Soleimani Rad J, et al. Bidirectional and opposite effects of naive mesenchymal stem cells on tumor growth and progression. Adv Pharm Bull. (2019) 9:539-58. doi: 10.15171/apb.2019.063

17. Zhang XL, Jiang C, Zhang ZX, Liu F, Zhang F, Cheng YF. The tumor-stroma ratio is an independent predictor for survival in nasopharyngeal cancer. Oncol Res Treat. (2014) 37:480-4. doi: 10.1159/000365165 
18. Chen Y, Zhang L, Liu W, Liu X. Prognostic significance of the tumorstroma ratio in epithelial ovarian cancer. Biomed Res Int. (2015) 2015:589301. doi: $10.1155 / 2015 / 589301$

19. Downey CL, Thygesen HH, Sharma N, Shaaban AM. Prognostic significance of tumour stroma ratio in inflammatory breast cancer. Springerplus. (2015) 4:68. doi: 10.1186/s40064-015-0852-7

20. Panayiotou H, Orsi NM, Thygesen HH, Wright AI, Winder M, Hutson R, et al. The prognostic significance of tumour-stroma ratio in endometrial carcinoma. BMC Cancer. (2015) 15:955. doi: 10.1186/s12885-015-1981-7

21. Xi KX, Wen YS, Zhu CM, Yu XY, Qin RQ, Zhang XW, et al. Tumorstroma ratio. (TSR) in non-small cell lung cancer. (NSCLC) patients after lung resection is a prognostic factor for survival. J Thorac Dis. (2017) 9:4017-26. doi: $10.21037 /$ jtd.2017.09.29

22. Hansen TF, Kjaer-Frifeldt S, Lindebjerg J, Rafaelsen SR, Jensen LH, Jakobsen A, et al. Tumor-stroma ratio predicts recurrence in patients with colon cancer treated with neoadjuvant chemotherapy. Acta Oncol. (2018) 57:528-33. doi: 10.1080/0284186X.2017.1385841

23. Zunder SM, Van Pelt GW, Gelderblom HJ, Mancao C, Putter H, Tollenaar RA, et al. Predictive potential of tumour-stroma ratio on benefit from adjuvant bevacizumab in high-risk stage II and stage III colon cancer. $\mathrm{Br} J$ Cancer. (2018) 119:164-9. doi: 10.1038/s41416-018-0083-0

24. Eriksen AC, Sorensen FB, Lindebjerg J, Hager H, Depont Christensen R, KjaerFrifeldt S, et al. The prognostic value of tumour stroma ratio and tumour budding in stage II colon cancer. A nationwide population-based study. Int J Colorectal Dis. (2018) 33:1115-24. doi: 10.1007/s00384-018-3076-9

25. Guinney J, Dienstmann R, Wang X, De Reynies A, Schlicker A, Soneson C, et al. The consensus molecular subtypes of colorectal cancer. Nat Med. (2015) 21:1350-6. doi: 10.1038/nm.3967

26. Owusu BY, Galemmo R, Janetka J, Klampfer L. Hepatocyte growth factor, a key tumor-promoting factor in the tumor microenvironment. Cancers. (2017) 9:35. doi: 10.3390/cancers9040035

27. Woolston A, Khan K, Spain G, Barber LJ, Griffiths B, Gonzalez-Exposito $\mathrm{R}$, et al. Genomic and transcriptomic determinants of therapy resistance and immune landscape evolution during anti-EGFR treatment in colorectal cancer. Cancer Cell. (2019) 36:35-50 e39. doi: 10.1016/j.ccell.2019.05.013

28. Labernadie A, Kato T, Brugues A, Serra-Picamal X, Derzsi S, Arwert E, et al. A mechanically active heterotypic E-cadherin/N-cadherin adhesion enables fibroblasts to drive cancer cell invasion. Nat Cell Biol. (2017) 19:224-37. doi: $10.1038 /$ ncb3478

29. Bercovici N, Guerin MV, Trautmann A, Donnadieu E. The remarkable plasticity of macrophages: a chance to fight cancer. Front Immunol. (2019) 10:1563. doi: 10.3389/fimmu.2019.01563

30. Hinshaw DC, Shevde LA. The tumor microenvironment innately modulates cancer progression. Cancer Res. (2019) 79:4557-66. doi: 10.1158/0008-5472.CAN-18-3962

31. Kalluri R. The biology and function of fibroblasts in cancer. Nat Rev Cancer. (2016) 16:582-98. doi: 10.1038/nrc.2016.73

32. Valkenburg KC, De Groot AE, Pienta KJ. Targeting the tumour stroma to improve cancer therapy. Nat Rev Clin Oncol. (2018) 15:366-81. doi: 10.1038/s41571-018-0007-1

33. Varkaris A, Gaur S, Parikh NU, Song JH, Dayyani F, Jin JK, et al. Ligandindependent activation of MET through IGF-1/IGF-1R signaling. Int J Cancer. (2013) 133:1536-46. doi: 10.1002/ijc.28169

34. Serao NV, Delfino KR, Southey BR, Beever JE, Rodriguez-Zas SL. Cell cycle and aging, morphogenesis, and response to stimuli genes are individualized biomarkers of glioblastoma progression and survival. BMC Med Genomics. (2011) 4:49. doi: 10.1186/1755-8794-4-49

35. Li H, Zhang SR, Xu HX, Wang Q, Li S, Li TJ, et al. SRPX2 and RAB31 are effective prognostic biomarkers in pancreatic cancer. J Cancer. (2019) 10:2670-8. doi: 10.7150/jca.32072

36. Jogi A, Vaapil M, Johansson M, Pahlman S. Cancer cell differentiation heterogeneity and aggressive behavior in solid tumors. Ups J Med Sci. (2012) 117:217-24. doi: 10.3109/03009734.2012.659294

37. Ishii G, Ochiai A, Neri S. Phenotypic and functional heterogeneity of cancer-associated fibroblast within the tumor microenvironment. Adv Drug Deliv Rev. (2016) 99:186-96. doi: 10.1016/j.addr.2015. 07.007
38. Lebleu VS, Kalluri R. A peek into cancer-associated fibroblasts: origins, functions and translational impact. Dis Model Mech. (2018) 11. doi: $10.1242 / \mathrm{dmm} .029447$

39. Ishibashi M, Neri S, Hashimoto H, Miyashita T, Yoshida T, Nakamura $\mathrm{Y}$, et al. CD200-positive cancer associated fibroblasts augment the sensitivity of Epidermal growth factor receptor mutation-positive lung adenocarcinomas to EGFR Tyrosine kinase inhibitors. Sci Rep. (2017) 7:46662. doi: 10.1038/srep46662

40. Su S, Chen J, Yao H, Liu J, Yu S, Lao L, et al. CD10(+)GPR77(+) cancerassociated fibroblasts promote cancer formation and chemoresistance by sustaining cancer stemness. Cell. (2018) 172:841-56 e816. doi: 10.1016/j.cell.2018.01.009

41. Yu Y, Xiao CH, Tan LD, Wang QS, Li XQ, Feng YM. Cancer-associated fibroblasts induce epithelial-mesenchymal transition of breast cancer cells through paracrine TGF-beta signalling. Br J Cancer. (2014) 110:724-32. doi: 10.1038/bjc. 2013.768

42. Li H, Batth IS, Qu X, Xu L, Song N, Wang R, et al. IGF-IR signaling in epithelial to mesenchymal transition and targeting IGF-IR therapy: overview and new insights. Mol Cancer. (2017) 16:6. doi: 10.1186/s12943-016-0576-5

43. Cheng Y, Song Y, Qu J, Che X, Song N, Fan Y, et al. The chemokine receptor CXCR4 and c-MET cooperatively promote epithelial-mesenchymal transition in gastric cancer cells. Transl Oncol. (2018) 11:487-97. doi: 10.1016/j.tranon.2018.02.002

44. Nakamura T, Nawa K, Ichihara A. Partial purification and characterization of hepatocyte growth factor from serum of hepatectomized rats. Biochem Biophys Res Commun. (1984) 122:1450-9. doi: 10.1016/0006-291X(84)91253-1

45. Stoker M, Gherardi E, Perryman M, Gray J. Scatter factor is a fibroblastderived modulator of epithelial cell mobility. Nature. (1987) 327:239-42. doi: $10.1038 / 327239 \mathrm{a} 0$

46. Gherardi E, Gray J, Stoker M, Perryman M, Furlong R. Purification of scatter factor, a fibroblast-derived basic protein that modulates epithelial interactions and movement. Proc Natl Acad Sci USA. (1989) 86:5844-8. doi: $10.1073 /$ pnas.86.15.5844

47. Wang R, Ferrell LD, Faouzi S, Maher JJ, Bishop JM. Activation of the Met receptor by cell attachment induces and sustains hepatocellular carcinomas in transgenic mice. J Cell Biol. (2001) 153:1023-34. doi: 10.1083/jcb.153.5.1023

48. Abounader R, Reznik T, Colantuoni C, Martinez-Murillo F, Rosen EM, Laterra J. Regulation of c-Met-dependent gene expression by PTEN. Oncogene. (2004) 23:9173-82. doi: 10.1038/sj.onc.1208146

49. Mitra AK, Sawada K, Tiwari P, Mui K, Gwin K, Lengyel E. Ligandindependent activation of c-Met by fibronectin and alpha(5)beta(1)-integrin regulates ovarian cancer invasion and metastasis. Oncogene. (2011) 30:156676. doi: 10.1038 /onc. 2010.532

50. Bertotti A, Comoglio PM, Trusolino L. Beta4 integrin is a transforming molecule that unleashes Met tyrosine kinase tumorigenesis. Cancer Res. (2005) 65:10674-9. doi: 10.1158/0008-5472.CAN-05-2827

51. Olaku V, Matzke A, Mitchell C, Hasenauer S, Sakkaravarthi A, Pace G, et al. c-Met recruits ICAM-1 as a coreceptor to compensate for the loss of CD44 in Cd44 null mice. Mol Biol Cell. (2011) 22:2777-86. doi: $10.1091 / \mathrm{mbc} . \mathrm{e} 11-02-0134$

52. Fischer OM, Giordano S, Comoglio PM, Ullrich A. Reactive oxygen species mediate Met receptor transactivation by $\mathrm{G}$ protein-coupled receptors and the epidermal growth factor receptor in human carcinoma cells. J Biol Chem. (2004) 279:28970-8. doi: 10.1074/jbc.M402508200

53. Pfeffer SR. Rab GTPases: master regulators that establish the secretory and endocytic pathways. Mol Biol Cell. (2017) 28:712-5. doi: $10.1091 / \mathrm{mbc}$.e16-10-0737

54. Chang YC, Su CY, Chen MH, Chen WS, Chen CL, Hsiao M. Secretory RAB GTPase 3C modulates IL6-STAT3 pathway to promote colon cancer metastasis and is associated with poor prognosis. Mol Cancer. (2017) 16:135. doi: 10.1186/s12943-017-0687-7

55. Wang G, Wei Z, Wu G. Role of Rab GTPases in the export trafficking of G protein-coupled receptors. Small GTPases. (2018) 9:130-5. doi: 10.1080/21541248.2016.1277000

56. Brozzi F, Diraison F, Lajus S, Rajatileka S, Philips T, Regazzi R, et al. Molecular mechanism of myosin Va recruitment to dense core secretory granules. Traffic. (2012) 13:54-69. doi: 10.1111/j.1600-0854.2011.01301.x 
57. Singh RK, Mizuno K, Wasmeier C, Wavre-Shapton ST, Recchi C, Catz $\mathrm{SD}$, et al. Distinct and opposing roles for Rab27a/Mlph/MyoVa and Rab27b/Munc13-4 in mast cell secretion. FEBS J. (2013) 280:892-903. doi: 10.1111/febs.12081

58. Yeo JC, Wall AA, Luo L, Stow JL. Rab31 and APPL2 enhance FcgammaR-mediated phagocytosis through PI3K/Akt signaling in macrophages. Mol Biol Cell. (2015) 26:952-65. doi: 10.1091/mbc.E14-101457

59. Baeza-Raja B, Li P, Le Moan N, Sachs BD, Schachtrup C, Davalos D, et al. p75 neurotrophin receptor regulates glucose homeostasis and insulin sensitivity. Proc Natl Acad Sci USA. (2012) 109:5838-43. doi: 10.1073/pnas.11036 38109

60. Chua CE, Tang BL. Engagement of the small GTPase Rab31 protein and its effector, early endosome antigen 1, is important for trafficking of the ligand-bound epidermal growth factor receptor from the early to the late endosome. J Biol Chem. (2014) 289:12375-89. doi: 10.1074/jbc.M114. 548321

Conflict of Interest: The authors declare that the research was conducted in the absence of any commercial or financial relationships that could be construed as a potential conflict of interest.

Copyright (c) 2020 Yang, Zhiheng, Zhanhuai, Qian, Yue, Xiaoxu, Jingsun, Shu and Kefeng. This is an open-access article distributed under the terms of the Creative Commons Attribution License (CC BY). The use, distribution or reproduction in other forums is permitted, provided the original author(s) and the copyright owner(s) are credited and that the original publication in this journal is cited, in accordance with accepted academic practice. No use, distribution or reproduction is permitted which does not comply with these terms. 